\title{
Fatores que interferem na não adesão de mulheres ao teste de Papanicolau: revisão integrativa
}

\author{
Factors that interfere with women's non-admission to the Papanicolaou test: \\ integrative review
}

\author{
Factores que interfieren en la no admisión de mujeres a la prueba de \\ Papanicolaou: revisión integrativa
}

\begin{abstract}
Débora Soares Gomes ${ }^{1 *}$, Jessyca Moreira Maciel${ }^{1}$, Sheron Maria Silva Santos ${ }^{1}$, Janayle Kéllen Duarte de Sales', Lívia Monteiro Rodrigues ${ }^{1}$, Rachel de Sá Barreto Luna Callou Cruz¹, Dayanne Rakelly de Oliveira ${ }^{1}$, Edilma Gomes Rocha Cavalcante ${ }^{1}$.
\end{abstract}

\section{RESUMO}

Objetivo: Identificar os fatores que interferem na não adesão de mulheres ao Teste de Papanicolau. Métodos: Trata-se de uma revisão integrativa, realizada de forma pareada nas bases de dados CINAHL, LILACS, MEDLINE e na biblioteca virtual Scielo, entre maio e agosto de 2021 por meio dos Descritores em Saúde Women, Papanicolau Test Uterine Cervical Neoplasms e Risk Factors, cruzados pelo operador booleano and. Selecionaram-se 19 artigos para amostra final. Resultados: Diversos fatores foram identificados como preditivos à não adesão do Teste de Papanicolau, dentre eles: baixa renda, escolaridade, faixa etária, falta de conhecimento sobre o teste, não possuir companheiro, uso de tabaco, álcool e outras drogas, vergonha ou medo do procedimento, acreditar estar bem de saúde, dificuldade de acesso aos serviços e falta de integralidade da assistência prestada. Considerações finais: Percebe-se que os fatores associados à não adesão do Teste de Papanicolau remetem a presença de iniquidades sociais e raciais, fatores individuais, condições e oferta dos serviços de saúde deficiente, além da cobertura insuficiente da realização do teste; sendo necessário, portanto, realizar ações educativas para sensibilizar a população sobre a imp ortância de realizar o exame para a detecção precoce, tratamento oportuno da doença e realização de busca ativa.

Palavras-chave: Mulheres, Teste de Papanicolau, Fatores de risco.

\section{ABSTRACT}

Objective: To identify the factors that interfere with women's non-adherence to the Papanicolaou test. Methods: This is an integrative review, carried out in a paired manner in the CINAHL, LILACS, MEDLINE databases and the Scielo virtual library, between May and August 2021 through the Descriptors in Women's Health, Papanicolaou Test Uterine Cervical Neoplasms and Risk Factors, crossed by the Boolean operator and. 19 articles were selected for the final sample. Results: Several factors were identified as predictors of non-adherence to the Pap smear, including: low income, education, age, lack of knowledge about the test, not having a partner, use of tobacco, alcohol and other drugs, shame or fear of the procedure, believing that they are in good health, difficulty in accessing services and lack of comprehensive care. Final considerations: It is noticed that the factors associated with non-adherence to the Pap smear test refer to the presence of social and racial inequalities, individual factors, conditions and provision of deficient health services, in addition to insufficient coverage of the test; therefore, it is necessary to carry out educational actions to sensitize the population about the importance of performing the exam for early detection, timely treatment of the disease and carrying out an active search.

Keywords: Women, Papanicolaou test, Risk factors.

\section{RESUMEN}

Objetivo: Identificar los factores que interfieren con la no adherencia de las mujeres a la prueba de Papanicolaou. Métodos: Se trata de una revisión integradora, realizada por parejas en las bases de datos CINAHL, LILACS, MEDLINE y en la biblioteca virtual Scielo, entre mayo y agosto de 2021 a través de los Descriptores en Salud de la Mujer, Prueba de Papanicolaou de Neoplasias Cervicales Uterinas y Factores de Riesgo, cruzados por el operador booleano y. Se seleccionaron 19 artículos para la muestra final. Resultados: Se identificaron varios factores como predictores de la no adherencia a la prueba de Papanicolaou, entre ellos: bajos ingresos, educación, edad, falta de conocimiento sobre la prueba, no tener pareja, uso de tabaco, alcohol y otras drogas, vergüenza o miedo. del procedimiento, por considerar que gozan de buena salud, dificultad para acceder a los servicios y falta de atención integral. Consideraciones finales: Se advierte que los factores asociados a la no adherencia a la prueba de Papanicolaou se refieren a la presencia de desigualdades sociales y raciales, factores individuales, condiciones y prestación de servicios de salud deficientes, además de cobertura insuficiente de la prueba; por tanto, es necesario realizar acciones educativa s para sensibilizar a la población sobre la importancia de realizar el examen para la detección precoz, el tratamiento oportuno de la enfermedad y la realización de una búsqueda activa.

Palabras clave: Mujeres, Prueba de Papanicolaou, Factores de riesgo.

${ }^{1}$ Universidade Regional do Cariri (URCA), Crato - CE. *E-mail: deboramahogany16@gmail.com

SUBMETIDO EM: 11/2021

ACEITO EM: 11/2021

PUBLICADO EM: 12/2021 


\section{INTRODUÇÃO}

O Câncer do Colo do Útero (CCU) é uma doença crônico-degenerativa, que representa um grave problema de saúde pública, devido sua alta prevalência, incidência, morbidade e mortalidade no Brasil (CARVALHO LRS e JURADO SR, 2018).

Segundo o Instituto Nacional de Câncer (INCA) (2021) o CCU é o terceiro tipo de câncer que mais acomete as brasileiras, com 16.710 casos novos diagnosticados somente no ano de 2020, atrás apenas do câncer de mama, cólon e reto. Nesse sentido, é importante realizar ações e intervenções para combater e controlar o $\mathrm{CCU}$, tendo como principal estratégia a detecção precoce das lesões precursoras através do Teste de Papanicolau (MINISTÉRIO DA SAÚDE, 2021; LEITE KNS, et al., 2018a).

O exame ou Teste de Papanicolau, também denominado exame citopatológico, esfregaço cervicovaginal ou colpocitologia oncótica cervical e popularmente conhecimento como exame de prevenção, diz respeito ao método de rastreamento de lesões precursoras do $\mathrm{CCU}$, bem como de sua detecção precoce. É indicado para pessoas do sexo feminino com idade entre 25 e 60 anos que tenham iniciado a vida sexual, devendo ser realizado anualmente e, somente trianual, após resultados negativos em dois exames consecutivos com intervalo de um ano entre eles (MINISTÉRIO DA SAÚDE, 2016).

Esse teste pode ser realizado pelo profissional enfermeiro ou pelo médico em estabelecimentos que permitam a segurança e a privacidade da mulher como, por exemplo, nas Unidades de Saúde da Família, Unidades Básicas de Saúde, ambulatórios especializados do Sistema Único de Saúde e em serviços privados (MACIEL LMA, et al., 2020).

No Brasil, o Teste de Papanicolau tem sido caracterizado como oportunista, tendo em vista basear-se principalmente na demanda espontânea da população, restringindo-se a mulheres que buscam os serviços de saúde, muitas vezes, por outro motivo, como também em períodos de campanhas contra o CCU e o câncer de mama (TIENSOLI S, et al., 2018).

Sob esse contexto, apesar do Teste de Papanicolau ser um método de rastreamento importante para o $\mathrm{CCU}$, ser prático, rápido, gratuito, indolor, quando executado da maneira correta, e o Ministério da Saúde, ao longo dos anos, venha instituindo metas e realizando ações para o combate e controle do CCU, o índice de mulheres que realizam o exame ainda é insatisfatório, pois, segundo dados divulgados pela Sociedade Brasileira de Oncologia (SBOC) (2018), 52\% das mulheres com idade indicada para realizar o teste, não o fazem, estando o Rio Grande do Norte (25\%), Roraima (27\%), Mato Grosso (27\%), Tocantins (27\%) e Bahia (29\%) com os índices mais baixos de realização do Papanicolau entre os estados brasileiros (MALTA EFGD, et al., 2017).

Diante do exposto, o presente trabalho objetivou identificar os fatores que interferem na não adesão de mulheres ao Teste de Papanicolau.

\section{MÉTODOS}

Trata-se de uma revisão integrativa da literatura construída conforme as seis etapas de Mendes KDS, et al. (2008), a saber: definição do tema e seleção da hipótese, delimitação dos critérios de inclusão e exclusão, definição das informações que serão extraídas, avaliação dos estudos, interpretação dos resultados e apresentação da revisão.

O estudo foi guiado pela pergunta norteadora: Quais os principais $f$ atores que interferem na não adesão de mulheres ao teste de rastreamento do CCU? Elaborada com base na estratégia Population, Variables and Outcomes (PVO) que guiou a busca dos artigos, onde $\mathrm{P}$ (population) corresponde a mulheres com idade entre 25 e 69 anos que já tiveram relação sexual, $\mathrm{V}$ (variable) corresponde exame de rastreamento do CCU e $\mathrm{O}$ (outcome) se refere aos fatores de risco que interferem na adesão a realização do teste (Quadro 1) (SILVA GA e OTTA E, 2014). 
Quadro 1 - Descritores de assunto localizados no MeSH para os componentes da pergunta de pesquisa segundo estratégia PVO.

\begin{tabular}{|c|c|c|}
\hline Itens da Estratégia & Componentes da pergunta & Descritores de assunto \\
\hline $\mathrm{P}$ & $\begin{array}{c}\text { Mulheres com idade entre 25 e 69 anos que já tiveram } \\
\text { relação sexual }\end{array}$ & Women \\
\hline $\mathrm{V}$ & Teste de rastreamento do CCU & $\begin{array}{c}\text { Papanicolaou Test Uterine } \\
\text { Cervical Neoplasms }\end{array}$ \\
\hline $\mathrm{O}$ & Fatores de risco que interferem na adesão a realização \\
do exame & Risk Factors \\
\hline
\end{tabular}

Fonte: Gomes DS, et al., 2021.

A busca de dados foi realizada durante os meses de maio a agosto de 2021 de forma pareada entre dois pesquisadores independentes que chegaram ao mesmo resultado utilizando o método de busca avançada e as categorias título, resumo e assunto. Desse modo, recuperou-se estudos indexados nas seguintes bases de dados eletrônicas: Cummulative Index to Nursing and Allied Health Literature (CINAHL), Literatura Latino americana e do Caribe em Ciências da Saúde (LILACS), Medical Literature Analysis and Retrievel System Online (MEDLINE) e biblioteca virtual Scientific Electronic Library Online (SciELO).

Para montar as chaves de busca foram utilizados os descritores controlados no Medical Subject Headings (MeSH) e Descritores em Ciências da Saúde (DeCS), em inglês: Women, Papanicolau Test, Uterine Cervical Neoplasms e Risk Factors. Combinados pelo operador booleano "and", ou utilizados separados de acordo com os critérios de cada base de dados; os descritores foram cruzados de forma simples (Women and Papanicolau Test and Uterine Cervical Neoplasms and Risk Factors) fenômeno que gerou um total de 606 estudos.

Para realizar a seleção dos artigos, primeiramente foi aplicado o filtro de texto completo disponível com o intuito de refinar a amostra obtida; depois foi realizada a leitura dos títulos e resumos das publicações. $O$ percurso percorrido na seleção, exclusão e inclusão dos artigos foi ilustrado em formato de figura para melhor detalhamento e compreensão das etapas realizadas (Figura 1).

Figura 1 - Fluxograma do processo de seleção dos estudos.

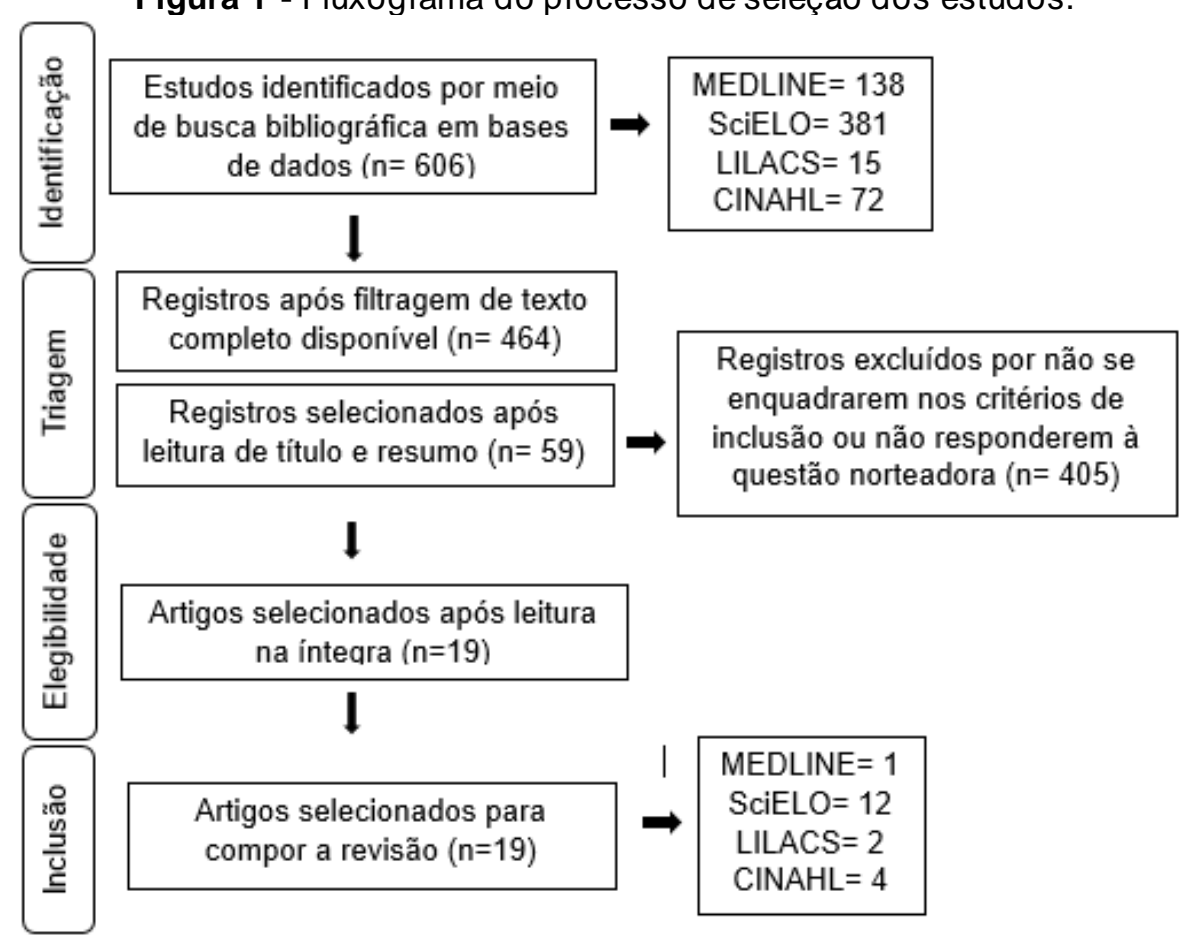

Fonte: Gomes DS, et al., 2021.

Dessa forma, elencaram-se como critérios de inclusão para a construção da presente revisão os fatores, a saber: ser artigo original publicado em periódico em formato de texto completo disponível de forma online e 
gratuita, bem como que seu conteúdo versasse sobre os fatores que interferem na não adesão do Teste de Papanicolau. Vale a pena ressaltar que não foram estabelecidas restrições quanto ao ano e idioma dos artigos, tendo em vista não haver marco histórico que justifique a análise por intervalo de tempo, bem como pelo critério relacionado ao idioma restringir artigos disponibilizados nos bancos de dados que possuam resultados significativos que contemplam o objetivo do estudo.

Destarte, durante o processo de seleção dos artigos excluíram-se todos os artigos duplicados já selecionados pelos critérios de inclusão, as produções científicas cujo tema não tinham relação com a proposta desta pesquisa, bem como, teses, dissertações, editoriais, carta ao editor e revisões integrativas.

No que diz respeito a análise e síntese dos artigos selecionados, foi utilizado um instrumento construído pelas próprias autoras para esse fim, que embora não seja validado, foi embasado no instrumento proposto porUrsi ES (2005), e contemplou os itens, a saber: autores, país que o estudo foi realizado, ano de publicação, objetivo do estudo, tipo de estudo, quantidade de participantes, principais fatores para não realização do Teste de Papanicolau e desfecho/conclusões evidenciadas. Os achados foram apresentados por meio de um quadro, de forma descritiva, no tópico resultados e interpretados de forma crítica onde foram confrontados com a literatura científica pertinente.

\section{RESULTADOS}

A partir da aplicação dos critérios de elegibilidade, 19 artigos foram selecionados para compor a amostra final e serem analisados para a construção da presente revisão (Quadro 2). Ao analisar os artigos, constatouse que os estudos foram publicados entre os anos 2003 e 2019, sendo 2018 o período com maior índice de publicação, perfazendo um total de $21 \%(n=4)$ dos estudos. Além disso, verificou-se que os artigos foram realizados em sítios distintos como, por exemplo, na Suécia, nos Estados Unidos, no Peru e na Estônia. Entretanto, os com maior predominância foram realizados no Brasil, 78,9\% ( $n=15)$.

Com relação aos objetivos do estudo, grande parte dos estudos tem foco na prevalência de realização exame citopatológico $37 \%(n=7)$, ou buscam nos seus objetivos conhecer os fatores que estão associados a não realização do exame $31,5 \%(n=6)$.

Os estudos apresentaram, números de participantes variados, com o menor número em um estudo qualitativo com 20 mulheres e maior em um estudo caso-controle de base populacional realizado com 266.706 mulheres de vários países. A maioria apresentou abordagem quantitativa $94,7 \%(n=18)$, sendo a maior parte do tipo transversal $78,8 \%(n=15)$ realizado na atenção primária a saúde.

Evidenciou-se que os principais motivos para a não realização do Teste de Papanicolau referem-se aos aspectos, a saber: baixa escolaridade, não possuir companheiro, baixa renda, mulheres mais jov ens e cor da pele não branca, bem como histórico de uso de tabaco, álcool e outras drogas, estado de desnutrição, desemprego, vergonha ou medo do procedimento, difícil acesso ao teste, acreditar estar bem de saúde, comportamento negativo de saúde, sofrer violência física ou sexualmente pelo companheiro, falta de conhecimento sobre o exame, nunca ter ido ou ir pouco a consulta de enfermagem/médica/ginecológica.

Dentre os principais fatores associados a não realização do teste, constatou-se que as causas permanecem ao longo dos anos (2003 a 2019, período dos estudos selecionados), estando a escolaridade, o estado civil, os extremos da faixa etária (mais jovens ou mais velhas), a renda, a cor da pele, o medo e a carência de conhecimento sobre o exame como os motivos pontuados pelos es tudos.

Quanto aos desfechos, parte dos estudos evidenciaram cobertura satisfatória de realização ao Teste de Papanicolau contudo considerada como sendo insuficiente para impactar no perfil epidemiológico do CCU. Ainda se percebe resistência de grupos específicos de mulheres para a realização do teste, com forte influência de iniquidades sociais. A realização do Papanicolau pelas gestantes também foi considerada insatisfatória, de forma que serviço não conseguiu oportunizar a realização do teste através da ida da mulher à consulta de pré-natal.

Outros aspectos verificados estão relacionados a falta de integralidade e à dificuldade de acesso das mulheres aos serviços de saúde. 
Quadro 2 - Distribuição dos artigos incluídos na revisão integrativa relativos aos autores, país e anos de publicação, tipo de estudo e número de participantes, fatores associados

à não adesão do exame citopatológico e conclusões dos autores.

\begin{tabular}{|c|c|c|c|c|}
\hline Autores (Ano) & Objetivo do estudo & $\begin{array}{l}\text { Tipo de estudo/ № } \\
\text { de participantes }\end{array}$ & Fatores associados à não adesão & Conclusões \\
\hline $\begin{array}{l}\text { Tiensoli SD, et } \\
\text { al. (2018) }\end{array}$ & $\begin{array}{l}\text { Estimar a prevalência do exame } \\
\text { Papanicolau e analisar fatores associados } \\
\text { à sua não realização. }\end{array}$ & $\begin{array}{l}\text { Estudo transversal } \\
\qquad 22.580\end{array}$ & $\begin{array}{l}\text { Baixa escolaridade, não possuir companheiro, } \\
\text { desnutrição, autoavaliação do estado de saúde } \\
\text { como negativo, possuir pelo menos um } \\
\text { comportamento negativo em saúde. }\end{array}$ & $\begin{array}{l}\text { Apesar da elevada cobertura do exame, ela ainda é } \\
\text { insatisfatória para alguns subgrupos de mulheres } \\
\text { que apresentam fatores de risco para não adesão. }\end{array}$ \\
\hline $\begin{array}{l}\text { Leite FMC, et } \\
\text { al. (2018) }\end{array}$ & $\begin{array}{l}\text { Analisar a associação entre a violência } \\
\text { por parceiro íntimo e a não realização do } \\
\text { exame citopatológico. }\end{array}$ & $\begin{array}{l}\text { Estudo transversal } \\
706\end{array}$ & $\begin{array}{l}\text { Baixa escolaridade, em união consensual, baixa } \\
\text { renda, fumantes e histórico de uso de drogas. }\end{array}$ & $\begin{array}{l}\text { Mulheres vítimas, física ou sexualmente, por seus } \\
\text { companheiros, estão mais vulneráveis a não } \\
\text { realização do exame de Papanicolau. }\end{array}$ \\
\hline $\begin{array}{l}\text { Broberg G, et } \\
\text { al. (2018) }\end{array}$ & 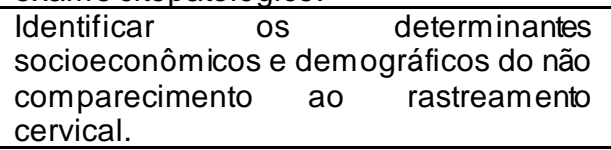 & $\begin{array}{l}\text { Estudo caso-controle } \\
\qquad 266.706\end{array}$ & $\begin{array}{l}\text { Baixa renda, baixa escolaridade e não coabitantes, } \\
\text { estar desempregada e receber benefícios da } \\
\text { previdência. }\end{array}$ & $\begin{array}{l}\text { O município de residência e os fatores } \\
\text { socioeconômicos estiveram fortemente associados à } \\
\text { menorfrequência ao rastreamento cervical. }\end{array}$ \\
\hline $\begin{array}{l}\text { Silva LSR, et } \\
\text { al. (2016) }\end{array}$ & $\begin{array}{l}\text { Investigar os motivos do não } \\
\text { comparecimento ao exame preventivo } \\
\text { Papanicolau. }\end{array}$ & $\begin{array}{l}\text { Estudo descritivo, } \\
\text { exploratório. } \\
39\end{array}$ & $\begin{array}{l}\text { Acreditar não precisar realizar o Papanicolau por } \\
\text { estarem bem de saúde. }\end{array}$ & $\begin{array}{l}\text { O conhecimento sobre os motivos do não } \\
\text { comparecimento ao exame, ajudará as equipes de } \\
\text { saúde no direcionamento das ações educativas com } \\
\text { toda população. }\end{array}$ \\
\hline $\begin{array}{l}\text { Oliveira PSD, } \\
\text { et al. (2016) }\end{array}$ & $\begin{array}{l}\text { Realizar intervenções de educação em } \\
\text { saúde após investigar a adesão das } \\
\text { mulheres ao exame de Papanicolau. }\end{array}$ & $\begin{array}{l}\text { Ensaio comunitário } \\
\qquad 60\end{array}$ & $\begin{array}{l}\text { Escolaridade relativamente baixa, não possuir } \\
\text { companheiro, falta de conhecimento sobre o exame, } \\
\text { medo ou vergonha do procedimento. }\end{array}$ & $\begin{array}{l}\text { Percebeu-se que as intervenções não aumentaram } \\
\text { a adesão ao exame. Mesmo com políticas públicas } \\
\text { voltadas para a população feminina do Brasil, ainda } \\
\text { existe resistência na realização do exame } \\
\text { Papanicolau. }\end{array}$ \\
\hline $\begin{array}{l}\text { Benard VB, et } \\
\text { al. }(2012)\end{array}$ & $\begin{array}{l}\text { Avaliar as tendências recentes na } \\
\text { incidência e mortalidade do câncer } \\
\text { cervical. }\end{array}$ & $\begin{array}{c}\text { Estudo transversal } \\
133.851\end{array}$ & $\begin{array}{l}\text { Não possuir plano de saúde e não possuir provedor } \\
\text { regular de saúde. }\end{array}$ & $\begin{array}{l}\text { A incidência de câncer cervical diminuiu, a } \\
\text { mortalidade permaneceu estável. }\end{array}$ \\
\hline $\begin{array}{l}\text { Kivistik } A \text {, et } \\
\text { al. }(2011)\end{array}$ & $\begin{array}{l}\text { Identificar o conhecimento dos fatores de } \\
\text { risco do câncer cervical. }\end{array}$ & $\begin{array}{l}\text { Estudo transversal } \\
1054\end{array}$ & $\begin{array}{l}\text { Visita recente a um ginecologista, medo de fazer o } \\
\text { exame Papanicolau, longas filas de consultas e } \\
\text { horários inadequados. }\end{array}$ & $\begin{array}{l}\text { As mulheres precisam de mais informações sobre os } \\
\text { fatores de risco do CCU e seu rastreamento. }\end{array}$ \\
\hline $\begin{array}{l}\text { Dias-da-Costa } \\
\text { JS, et al. } \\
(2019) \\
\end{array}$ & $\begin{array}{l}\text { Estimar a prevalência de exame } \\
\text { citopatológico não realizado nos últimos } \\
\text { três anos e de nunca realizado em } \\
\text { mulheres,e analisarfatores associados }\end{array}$ & $\begin{array}{c}\text { Estudo transversal } \\
1.128 \\
\end{array}$ & $\begin{array}{l}\text { Baixa renda, mais novas, nunca se consultaram com } \\
\text { médico e baixa escolaridade. }\end{array}$ & $\begin{array}{l}\text { A cobertura do exame citopatológico é alta, poucas } \\
\text { mulheres em atraso e sem nunca o ter realizado, } \\
\text { foram influenciadas por iniquidades sociais. }\end{array}$ \\
\hline $\begin{array}{l}\text { Muller DK, et } \\
\text { al. (2008) }\end{array}$ & $\begin{array}{l}\text { Verificar a cobertura de realização do } \\
\text { exame preventivo de CCU e os fatores } \\
\text { associados na população de mulheres de } \\
20 \text { a } 60 \text { anos. }\end{array}$ & $\begin{array}{l}\text { Estudo transversal } \\
\qquad 867\end{array}$ & $\begin{array}{l}\text { Renda e escolaridade baixas, mulheres jovens e as } \\
\text { mais velhas, não brancas, não possuir companheiro. }\end{array}$ & $\begin{array}{l}\text { Apesar da cobertura de realização do exame } \\
\text { Papanicolau ser elevada, ainda existem vários } \\
\text { fatores associados à iniquidade e falta de } \\
\text { integralidade da assistência. }\end{array}$ \\
\hline $\begin{array}{l}\text { Ribeiro L, et } \\
\text { al. (2016) }\end{array}$ & $\begin{array}{l}\text { Estimara prevalência e identificar fatores } \\
\text { associados à não realização do exame } \\
\text { citopatológico do colo do útero entre } \\
\text { mulheres que frequentaram o pré-natal. }\end{array}$ & $\begin{array}{l}\text { Estudo transversal } \\
\qquad 308\end{array}$ & $\begin{array}{l}\text { Sentir-se saudável e não perceber necessidade de } \\
\text { cuidados de saúde, solteiras, jovens e baixa } \\
\text { escolaridade. }\end{array}$ & $\begin{array}{l}\text { O contato com o serviço de saúde para realização do } \\
\text { pré-natal não contribuiu para aumentar o acesso ao } \\
\text { exame, demonstrando perda de oportunidade. }\end{array}$ \\
\hline
\end{tabular}




\begin{tabular}{|c|c|c|c|c|}
\hline Autores (Ano) & Objetivo do estudo & $\begin{array}{l}\text { Tipo de estudo/ № } \\
\text { de participantes }\end{array}$ & Fatores associados à não adesão & Conclusões \\
\hline $\begin{array}{l}\text { Oliveira MV, et } \\
\quad \text { al. (2014) }\end{array}$ & $\begin{array}{l}\text { Analisar os fatores associados a não } \\
\text { realização do exame de Papanicolau } \\
\text { entre as mulheres quilombolas. }\end{array}$ & $\begin{array}{l}\text { Estudo transversal } \\
\qquad 348\end{array}$ & $\begin{array}{l}\text { Mais jovens ou idade superior a } 50 \text { anos, baixa } \\
\text { escolaridade, sem companheiro, nunca ter feito o } \\
\text { exame clínico das mamas, ou tê-lo executado há um } \\
\text { tempo maior ou igual a } 3 \text { anos, atendimento à saúde } \\
\text { em diferentes unidades. }\end{array}$ & $\begin{array}{l}\text { Os achados possivelmente refletem a falta de } \\
\text { integralidade dos serviços de saúde disponíveis para } \\
\text { a população quilombola, com dificuldades de acesso } \\
\text { em saúde. }\end{array}$ \\
\hline $\begin{array}{l}\text { Terlan RJ, et } \\
\text { al. (2018) }\end{array}$ & $\begin{array}{l}\text { Medir a prevalência e identificar fatores } \\
\text { associados a não realização de exame } \\
\text { citopatológico de colo uterino entre } \\
\text { gestantes. }\end{array}$ & $\begin{array}{l}\text { Estudo transversal } \\
\qquad 1.474\end{array}$ & $\begin{array}{l}\text { Baixa escolaridade, aborto prévio, consumo de } \\
\text { álcool durante a gestação e baixo número de } \\
\text { consultas de pré-natal. }\end{array}$ & $\begin{array}{l}\text { Proporção expressiva das gestantes não realizou o } \\
\text { exame citopatológico. }\end{array}$ \\
\hline $\begin{array}{l}\text { Brischiliari } \\
\text { SCR, et al. } \\
\quad(2012)\end{array}$ & $\begin{array}{l}\text { Analisar os fatores de risco relacionados } \\
\text { à não realização do exame de } \\
\text { Papanicolau entre as mulheres que } \\
\text { vivenciam a menopausa. }\end{array}$ & $\begin{array}{l}\text { Estudo transversal } \\
\qquad 456\end{array}$ & $\begin{array}{l}\text { Menor escolaridade, cor não branca, baixa renda, } \\
\text { ocupação não remunerada. }\end{array}$ & $\begin{array}{l}\text { Melhorar o conhecimento das mulheres e diminuir } \\
\text { fatores para a não realização do exame de rastreio } \\
\text { do CCU. }\end{array}$ \\
\hline $\begin{array}{l}\text { Cesar JA, et } \\
\text { al. (2003) }\end{array}$ & $\begin{array}{l}\text { Conhecer a prevalência de não realização } \\
\text { de exame citopatológico e identificar } \\
\text { possíveis fatores associados à sua não } \\
\text { realização. }\end{array}$ & $\begin{array}{l}\text { Estudo transversal } \\
\qquad 1.302\end{array}$ & $\begin{array}{l}\text { Cor parda ou preta, de menoridade, renda familiare } \\
\text { escolaridade baixas, sem companheiro e que } \\
\text { tiveram o primeiro parto com } 25 \text { anos ou mais de } \\
\text { idade. }\end{array}$ & $\begin{array}{l}\text { O serviço de detecção precoce do CCU mostrou-se } \\
\text { pouco efetivo por cobrir menos da metade das } \\
\text { mulheres em idade fértil e desigual devido o acesso } \\
\text { ao exame variar de acordo com as características } \\
\text { das mulheres. }\end{array}$ \\
\hline $\begin{array}{l}\text { Cesar JA, et } \\
\text { al. (2012) }\end{array}$ & $\begin{array}{l}\text { Determinar a prevalência e identificar } \\
\text { fatores associados ao não rastreamento } \\
\text { voluntário para citopatológico de colo } \\
\text { uterino entre puérperas em Rio Grande } \\
\text { (RS). }\end{array}$ & $\begin{array}{l}\text { Estudo transversal } \\
2.288\end{array}$ & $\begin{array}{l}\text { Desconhecer a necessidade de realizar o exame, } \\
\text { medo ou vergonha, mulheres jovens, renda e } \\
\text { escolaridade baixas, sem companheiro, fumantes, } \\
\text { gravidez não planejada e poucas consultas pré- } \\
\text { natal. }\end{array}$ & $\begin{array}{l}\text { Quanto maior o risco para CCU, menor a chance de } \\
\text { a gestante realizar o exame de rastreio do CCU. }\end{array}$ \\
\hline $\begin{array}{l}\text { Albuquerque } \\
\text { KM, et al. } \\
\text { (2009) }\end{array}$ & $\begin{array}{l}\text { Avaliar a cobertura real do teste de } \\
\text { Papanicolau, destacando os fatores } \\
\text { associados à não-realização. }\end{array}$ & $\begin{array}{l}\text { Estudo transversal } \\
258 \\
\end{array}$ & $\begin{array}{l}\text { Viver sem companheiro, não ter dado à luze não ter } \\
\text { realizado consulta médica no último ano, baixa } \\
\text { escolaridade. }\end{array}$ & $\begin{array}{l}\text { A cobertura do Papanicolau foi maior do que } 60 \% \text {, } \\
\text { sendo considerada satisfatória, porém insuficiente } \\
\text { para impactar no perfil epidemiológico do CCU. }\end{array}$ \\
\hline $\begin{array}{l}\text { Aldave- } \\
\text { Zamora A, et } \\
\text { al. }(2019)\end{array}$ & $\begin{array}{l}\text { Determinar os fatores associados à não } \\
\text { adesão ao rastreio atempado com } \\
\text { Papanicolau na detecção precoce do } \\
\text { cancro do colo do útero. }\end{array}$ & $\begin{array}{l}\text { Estudo transversal } \\
130 \\
\end{array}$ & $\begin{array}{l}\text { Mais jovens, nível de escolaridade superior, } \\
\text { percepções de suscetibilidade ao câncer, barreiras } \\
\text { para realizar o exame. }\end{array}$ & $\begin{array}{l}\text { Há uma elevada frequência de não adesão ao } \\
\text { Papanicolau, as quais estão associadas } \\
\text { principalmente às percepções sobre o CCU. }\end{array}$ \\
\hline $\begin{array}{c}\text { Ferreira } \\
\text { MLSM. (2009) }\end{array}$ & $\begin{array}{l}\text { Analisar os motivos que influenciaram um } \\
\text { grupo de mulheres a nunca ter realizadoo } \\
\text { exame de Papanicolau mesmo após } \\
\text { iniciarem a atividade sexual. }\end{array}$ & $\begin{array}{l}\text { Estudo qualitativo } \\
\qquad 20\end{array}$ & $\begin{array}{l}\text { Desconhecimento sobre a doença e o exame, medo } \\
\text { de realizar o exame e do resultado, vergonha de se } \\
\text { submeter ao exame, dificuldade de acesso, cultura } \\
\text { biomédica e não preventiva. }\end{array}$ & $\begin{array}{l}\text { O comportamento e atitudes das mulheres pode } \\
\text { torná-las vulneráveis à doença, alguns fatores são } \\
\text { obstáculos para prevenção do CCU. }\end{array}$ \\
\hline $\begin{array}{l}\text { Amorim } \\
\text { VMSL, et al. } \\
(2006)\end{array}$ & $\begin{array}{l}\text { Analisar a prevalência da não realização } \\
\text { do exame de Papanicolau segundo } \\
\text { variáveis socioeconômicas, demográficas } \\
\text { e de comportamentos relacionados à } \\
\text { saúde. }\end{array}$ & $\begin{array}{l}\text { Estudo transversal } \\
\qquad 290\end{array}$ & $\begin{array}{l}\text { Ter de } 40 \text { a } 59 \text { anos, não branca, baixa escolaridade. } \\
\text { Acreditar ser desnecessário, vergonha, dificuldades } \\
\text { referentes aos serviços de saúde. }\end{array}$ & $\begin{array}{l}\text { Foram evidenciadas desigualdades } \\
\text { socioeconômicas e raciais elevadas quanto à } \\
\text { realização do exame de Papanicolau. }\end{array}$ \\
\hline
\end{tabular}




\section{DISCUSSÃo}

Através dos estudos selecionados, foi possível perceber que a não adesão de mulheres ao Teste de Papanicolau se trata de um problema antigo de saúde pública, uma vez que foram encontrados estudos realizados há praticamente dez anos versando sobre a temática e, por mais que haja considerações feitas pelos pesquisadores como, por exemplo, a realização de busca ativa e a educação em saúde para o empoderamento das mulheres e que as políticas públicas voltadas à saúde da mulher tenham sido aprimoradas, a adesão as mulheres ao exame ainda é precária estando suas causas associada a diversos fatores que passam por questões individuais e coletivas, com destaque para os fatores sociais, econômicos e raciais, que demonstram iniquidades em saúde (CESAR JA, et al., 2003; OLIVEIRA PSD, et al., 2016).

Um estudo realizado em 2015 que entrevistou 1.128 mulheres da zona urbana de São Leopoldo-RS a respeito dos fatores associados a não realização do Teste de Papanicolau, embora tenha identificado cobertura satisfatória do exame no município, havendo poucas mulheres que nunca o tenha realizado ou que estivessem com sua realização pendente, concluiu que as iniquidades sociais dificultam o acesso ao teste e influenciam na não adesão de mulheres à realização do Papanicolau (DIAS-DA-COSTA JS, et al., 2019).

Dessa forma, grande parte dos estudos sinalizaram como importantes preditores da não adesão ao teste, a baixa escolaridade, a baixa renda familiar e a cor da pele não branca. Um estudo realizado com mulheres do sexo, em uma capital do nordeste brasileiro, mostrou resultados semelhantes, com cobertura insatisfatória do Teste de Papanicolau para o subgrupo de mulheres com ensino fundamental incompleto, baixa renda e cor da pele preta/parda (MAGALHAES RLB, et al., 2019).

Os resultados desse estudo demonstram que mulheres que vivem sem companheiro realizam menos 0 exame. Corroborando com esses dados, estudos mostraram que mulheres casadas ou em união estável são mais propensas de serem rastreadas (TIENSOLI S, et al., 2018; MOREIRA APL e CARVALHO AT, 2020). Em geral, esses achados são entendidos a partir de uma relação direta entre a vida sexual e outras demandas que levam a mulher às unidades de saúde, como pré-natal e planejamento familiar, o que favorece a realização do exame (RIBEIRO, et al., 2016).

Entretanto, um estudo concluiu que a realização do Teste de Papanicolau durante o acompanhamento pré-natal também foiconsiderada insatisfatória, uma vez que ainda existem lacunas do rastreio em gestantes, demanda que exige capacitação dos profissionais e adequação dos serviços de saúde para oportunizar a realização do Teste de Papanicolau, tendo em vista já ter sido evidenciado que a sua realização durante o período inicial da gravidez é expressamente eficaz na detecção precoce do CCU (GASPARIN VA, et al., 2020; SEKINE M, et al., 2018).

Outro fator evidenciado foi a baixa cobertura do exame nas mulheres com idades jovens. Resultado que confirma esse achado foi encontrado em estudo realizado na capital da Paraíba, Brasil, que apresentou maior prevalência de adesão ao exame em mulheres com maior faixa etária, de 35 anos acima (MOREIRA APL e CARVALHO AT, 2020).

Fatores comportamentais de saúde como histórico de uso de tabaco, álcool e outras drogas, também foram associados a não realização do Teste de Papanicolau. No entanto, estudo realizado em todos os estados do Brasil, demonstrou predomínio de mulheres que não abusavam do álcool (89,8\%) dentre as que não realizaram o rastreio (TIENSOLI S, et al., 2018).

Vale apena ressaltar que fatores comportamentais e de estilo de vida não saudáveis, como não praticar atividade física, alimentação desequilibrada, tabagismo e alto consumo de álcool estão associados a mai or risco para o desenvolvimento dos diversos tipos de câncer (MINISTÉRIO DA SAÚDE, 2021).

A vergonha e o medo também foram associados à não participação no rastreamento do CCU. Concordando com estes resultados, outros estudos apresentaram que os sentimentos vivenciados pelas mulheres podem estar associados ao medo/vergonha do procedimento, medo do profissional ou mesmo do resultado, interferindo na adesão ao teste (CHANG HK, et al., 2017; SILVA JP, et al., 2018; RAFAEL RMR e MOURA ATMS, 2017). 
O medo pode ser relacionado ao relato de outras pessoas sobre a realização do exame, que surge junto com o medo da dor e de um possível resultado positivo para o CCU. E nesse sentido, associado a esses sentimentos, muitas mulheres acabam adiando sua realização e culminam não executando-o (CARVALHO LRS e JURADO SR, 2018).

Também foram identificados nos estudos como fatores a não adesão ao teste, à falta de conhecimento sobre o Papanicolau, acreditar estar bem de saúde, não havendo necessidade de realizar o teste e acompanhamento em saúde deficiente. Outros estudos demonstram que a falta de informação da população sobre a necessidade e importância do teste de Papanicolau é um dos problemas críticos à sua adesão (DANTAS PVJ, et al., 2018; GRANDO AS, et al., 2017; IGLESIAS GA, et al., 2019).

Sob este aspecto, um estudo que avaliou o conhecimento e a prática de 320 mulheres atendidas em três Unidades de Saúde da Família (USF) do município Caxias-MA em 2018 sobre o Teste de Papanicolau, constataram que $72,8 \%$ das entrevistadas possuam conhecimento inadequado a respeito do teste no que diz respeito a sua finalidade, o material coletado e os cuidados que precedem a realização da coleta do material cervical (SILVA LA, et al., 2021).

Segundo os autores, este achado é relevante de ser apreciado, uma vez que o teste diz respeito a um método de diagnóstico precoce ao CCU que elava, por sua vez, as chances de cura e minimiza os índices de evolução para casos graves, não prevenindo, portanto, a ocorrência do $\mathrm{CCU} \mathrm{e}$, assim, as mulheres que realizam o Papanicolau de forma periódica podem desenvolver CCU (SILVA LA, et al., 2021).

Neste sentido, constata-se que o conhecimento a respeito do Teste de Papanicolau está diretamente relacionado com o perfil sociodemográficos apresentado pela mulher. Um estudo realizado com 180 mulheres que residem no interior de São Paulo que buscou conhecer as causas que estão relacionadas com o conhecimento sobre o Teste de Papanicolau, constatou que das participantes que apresentaram conhecimento insatisfatório, 33,3\% eram de cor não branca, 35,6\% possuíam menos que dois salários mínimos e 30,8\% tinham menos que 9 anos de estudo. Após análise comparativa dos dados, os autores chegaram à conclusão de que mulheres de cor branca, com renda familiar acima de 2 salários mínimos e sem queixa ginecológica, apresentaram conhecimento satisfatório acerca do teste (SOARES MBO, et al., 2020).

Aspectos referentes as condições do próprio serviço de saúde também foram destacadas, como a falta de integralidade e acesso deficiente. Estes resultados corroboram com estudo realizado no estado do Amazonas, onde falhas na prestação da assistência e dificuldades de acesso ao serviço de saúde foram apontados como motivos para as mulheres não terem realizado o Teste de Papanicolau (VIANA JN, et al., 2019).

Tais evidências sinalizam desvio das funções da atenção primária a saúde, responsável por garantir o direito à atenção integral à saúde da população, de forma resolutiva e de qualidade, por meio de ações de promoção da saúde e prevenção do CCU, através da realização do teste de rastreamento, educação em saúde e vacinação da população-alvo recomendada (MINISTÉRIO DA SAÚDE, 2016).

Diante dos resultados expostos constata-se que a adesão ao exame Papanicolau ainda é baixa em algumas regiões e para alguns grupos populacionais específicos, e que as mulheres precisam ser empoderadas sobre a importância do teste, tendo em vista possuírem carência de informações ou pensamentos equivocados a respeito do rastreamento e prevenção contra o CCU (GRANDO AS, et al., 2017; DANTAS PVJ, et al., 2018; IGLESIAS GA, et al., 2019; MOREIRA APL e CARVALHO AT, 2020).

Destaca-se a importância de os profissionais conhecerem os fatores associados à não adesão do exame citopatológico e disponibilizarem informações para a população, por meio da educação em saúde, na perspectiva de esclarecer as dúvidas e minimizar os tabus e medos existentes, além de evidenciar que mesmo não tendo parceiro ou aparentemente estando bem de saúde é importante realizar o Teste de Papanicolau para o diagnóstico precoce de lesões preditivas ao CCU e realização de tratamento oportuno (CHANG HK, et al., 2017; SILVA JP, et al., 2018; RAFAEL RMR e MOURA ATMS, 2017). 


\section{CONSIDERAÇÕES FINAIS}

Verificou-se que os principais fatores associados à não adesão do Teste de Papanicolau remetem a presença de iniquidades sociais e raciais, fatores individuais e/ou comportamentais e condições dos serviços de saúde, causas pontuadas há praticamente dez anos e que permanecem ao longo dos anos, fazendo-se perceber a necessidade de realizar ações educativas para sensibilizar a população sobre a importância do teste de rastreamento para a detecção precoce e tratamento oportuno do $\mathrm{CCU}$, bem como a prática de busca ativa para fornecer maior alcance da cobertura de mulheres em idade-alvo, adequando as ações em saúde a realidade da população que se deseja atingir, além de avaliar o alcance das ações e/ou intervenções realizadas, constatando a necessidade de mudanças ou adaptações, para minimizar e/ou solucionar à não adesão ao Teste de Papanicolau.

\section{REFERÊNCIAS}

1. ALBUQUERQUE KM, et al. Pap smear coverage and factors associated with non-participation in cervical cancer screening: an analysis of the Cervical Cancer Prevention Program in Pernambuco State, Brazil. Cadernos de saude publica, 2009;25(2): 301-309.

2. ALDAVE-ZAMORA A, APOLAYA-SEGURA M. Factores asociados al incumplimiento del despistaje oportuno con papanicolaou en la detección tem prana del cáncer de cérvix en un centro poblado rural. Acta Médica Peruana, 2019; 36(4): 259-266.

3. AMORIM VMSL, et al. Factors associated with women's failure to submit to Pap smears: a population-based study in Campinas, São Paulo, Brazil. Cadernos de saúde pública, 2006;22(11):2329-2338.

4. BARBOSA GSL, et al. Realização do exame citopatológico em mulheres: uma revisão integrativa. Research, Society and Development, 2020;9(11):e2339119006.

5. BENARD VB, et al. Vital signs: cervical cancer incidence, mortality, and screening-United States, 2007-2012. Morbidity and mortality weekly report, 2014;63(44):1004.

6. BRISCHILIARI SC, et al. Factors associated with lack of Pap smear screening in a group of postmenopausal Brazilian women. Cadernos de saúde pública, 2012;28(10):1976-1984.

7. BROBERG G, et al. Determinantes socioeconômicos e demográficos que afetam a participação no programa de rastreamento cervical sueco: Um estudo de caso-controle de base populacional. PLoS ONE, 2018; 13(1): e0190171.

8. CARVALHO LRS, JURADO SR. Motivos que influenciam a não realização do exame de Papanicolau. 2018. Revista Científica de Enfermagem (RECIEN), 2018; 8(23):39-46.

9. CESAR JA, et al. Factors associated with non-participation in screening for cervical cancer in Southem Brazil. Cadernos de saude publica, 2003;19(5): 1365-1372.

10. CESAR JA, et al. Pap smears among pregnant women in Southern Brazil: a representative cross-sectional survey. Revista Brasileira de Ginecologia e Obstetrícia, 2012;34(11): 518-523.

11. CHANG HK, et al. Factors associated with participation in cervical cancer screening among young Koreans: a nationwide cross-sectional study. BMJ open, 2017; 7(4): e013868.

12. DANTAS PVJ, et al. Conhecimento das mulheres e fatores da não adesão acerca do exame papanicolau. Rev enferm UFPE online, 2018;12(3): 684-91.

13. DIAS-DA-COSTA JS, et al. Fatores associados a não realização de exame citopatológico em São Leopoldo, Rio Grande do Sul, 2015: estudo transversal de base populacional. Epidemiologia e Serviços de Saúde, 2019; 28: e2018203.

14. FERREIRA MLS, et al. Influence reasons that inhibit women from doing papanicolaou test. Escola Anna Nery, 2009; 13(2): 378-384.

15. GASPARIN VA, et al. Rastreamento do câncer de colo do útero durante o acompanhamento pré-natal. Rev. Eletr. Enferm. [Internet]., 2020;22: 63482.

16. GRANDO AS, et al. Conhecimento e prática do exame citopatológico de colo uterino entre acadêmicas de diferentes áreas. Rev. enferm. UFPE online, 2017;11(8): 3206-3213.

17. IGLESIAS GA, et al. Conhecimento e adesão ao Papanicolau de mulheres de uma rede de atenção primária à saúde. Rev Ciênc Med., 2019;28(1): 21-30.

18. MINISTÉRIO DA SAÚDE. Instituto Nacional de Câncer José Alencar Gomes da Silva (INCA). Detecção precoce do câncer. Instituto Nacional de Câncer José Alencar Gomes da Silva. 2021. Disponível em: https://www.inca.gov.br/sites/ufu.sti.inca.local/files//media/document//deteccao-precoce-do-cancer.pdf. Acessado em: 10 de novembro de 2021.

19. KIVISTIK A, et al. Women's knowledge about cervical cancer risk factors, screening, and reasons for non-participation in cervical cancer screening programme in Estonia. BMC women's health, 2011;11(1):1-6.

20. LEITE FMC, et al. Implicações das violências contra as mulheres sobre a não realização do exame citopatológico. Revista de Saúde Pública, 2018b;52: 89.

21. LEITE KNS, et al. Exame Papanicolau: fatores que influenciam a não realização do exame em mulheres de 40 a 65 anos. Arquivos de Ciências da Saúde, 2018a;25(2): 15-19. 
22. MACIEL LMA, et al. A importância do exame papanicolau realizado pelo enfermeiro para o diagnóstico do câncer no colo uterino. Revista Brasileira Interdisciplinar de Saúde, 2020;2(2): 88-92.

23. MAGALHAES RLB, et al. Fatores associados à realização do exame citopatológico em mulheres profissionais do sexo. Rev. baiana enferm., 2018;32: e2593.

24. MALTA EFGD, et al. Prática inadequada de mulheres acerca do Papanicolau. Texto Contexto Enferm, 2017; 26(1): e5050015.

25. MENDES KDS, et al. Revisão integrativa: método de pesquisa para a incorporação de evidências na saúde e na enfermagem. Texto contexto - enferm., 2008;17(4): 758-764.

26. MINISTÉRIO DA SAÚDE. Instituto Nacional de Câncer José Alencar Gomes da Silva (INCA). Diretrizes brasileiras para o rastreamento do câncer do colo do útero. Coordenação de Prevenção e Vigilância. Divisão de Detecção Precoce e Apoio à Organização de Rede. 2016. Disponível em: https://www.inca.gov.br/sites/ufu.sti.inca.local/files/media/document/diretrizesparaorastreamentodocancerdocolodou tero_2016_corrigido.pdf. Acessado em:1 de outubro de 2021.

27. MOREIRA APL, CARVALHO AT. Tendência de Realização da Citologia Oncótica e Fatores Associados em Mulheres de 25 a 64 anos. Rev. bras. ciênc. saúde, 2020;24(1): 17-28.

28. MULLER DK, et al. Cobertura do exame citopatológico do colo do útero na cidade de São Leopoldo, Rio Grande do Sul, Brasil. Cadernos de Saúde Pública, 2008;24: 2511-2520.

29. OLIVEIRA MV, et al. Fatores associados a não realização de Papanicolau em mulheres quilombolas. Ciência \& Saúde Coletiva, 2014;19(11): 4535-4544.

30. OLIVEIRA PSD, et al. Adesão das mulheres ao exame preventivo de câncer de colo de útero: um ensaio comunitário. Rev. Enferm. Ufpe Online, 2016;2(10):442-448.

31. RAFAEL RMR, MOURA ATMS. Modelo de Crenças em Saúde e o rastreio do câncer do colo uterino: avaliando vulnerabilidades. Enfermagem Uerj, 2017;25(1):NA-NA.

32. RIBEIRO L, etal. Opportunistic screening versus missed opportunities: non-adherence to Pap smear testing in women attending prenatal care. Cadernos de saude publica, 2016;32(6):1-8.

33. SEKINE M, et al. Malignancy during pregnancy in Japan: an exceptional opportunity for early diagnosis. BMC pregnancy and childbirth, 2018;18(1): 1-5.

34. SILVA GA, OTTA E. Revisão sistemática e meta-análise de estudos observacionais em Psicologia. Revista Costarricense de Psicología, 2014; 33: 137-153.

35. SILVA JP, et al. Papanicolau exam: factors that influence the failure to perform the examination in women aged to 40 65 years. Arq. Ciênc. Saúde, 2018;25(2): 15-9.

36. SILVA LA, et al. Conhecimento e prática de mulheres atendidas na atenção primária a saúde sobre o exame Papanicolau. R. pesq.: cuid. fundam. Online, 2021;13:1013-1019.

37. SOCIEDADE BRASILEIRA DE ONCOLOGIA (SBOC). SBOC divulga que $52 \%$ não fazem exame ginecológico preventivo de câncer. 2018. Disponível em: https://sboc.org.br/noticias/item/1151-sboc-divulga-que-52-nao-fazemexame-ginecologico-preventivo-de-cancer. Acesso em:3 de novembro de 2021.

38. TERLAN RJ, CESAR JA. Não realização de citopatológico de colo uterino entre gestantes no extremo sul do Brasil: prevalência e fatores associados. Ciência \& Saúde Coletiva, 2018;23(11):3557-3566.

39. TIENSOLI S, et al. Avaliação da não realização do exame Papanicolau por meio do Sistema de Vigilância por inquérito telefônico. Revista da Escola de Enfermagem da USP, 2018; 52: e03390.

40. VIANA JN, et al. Determinantes sociais da saúde e prevenção secundária do câncer do colo do útero no Estado do Amazonas, Brasil. Medicina (Ribeirão Preto, online), 2019;52(2): 110-20. 